\title{
EXPERIMENTAL STUDY OF MAGNUS EFFECT OVER AN AIRCRAFT WING
}

\author{
Kavithasan Patkunam ${ }^{1}$, Samay Sigamani ${ }^{2}$, Pedaballi Mahathi ${ }^{3}$, Selvakumaran. $\mathbf{T}^{4}$ \\ ${ }^{1,2,3}$ B.Tech Aerospace Engineering Graduate, Aerospace Engineering, SRM University, Tamil Nadu, India \\ ${ }^{4}$ Assistant Professor (Senior Grade), Aerospace Engineering, SRM University, Tamil Nadu, India
}

\begin{abstract}
We present this paper as an experimental study of employing Magnus Effect in an aircraft wing. The scope and interest of study of Magnus Effect and its application in Aerospace Engineering is widely increasing all over the world to make use of the Magnus Force for enhanced Lift by Drag Ratio. Most of the trials weren't successful due to the heavy Drag Force produced by large cylinders, even though sufficient Lift Force was generated. The main stereotypical apprehension of Magnus Effect is that it could be applied only over symmetrical bodies like a spinning ball(sphere), or a cylinder or a disc. We suggest an idea to be applied differently by maintaining a constant circumferential speed over an airfoil profile. We propose to initiate the project work by considering a symmetrical airfoil and study it's aerodynamic characteristics and make an attempt in extending it's performing envelope by implementing Magnus Effect. Our intention is to provide the constant circumferential speed over the airfoil skin is by compositing a treadmill like motion contributed by a series of rollers fastened over a chain track and driven by a stepper motor. The Rollers coupled Treadmill spins in a clockwise direction, when air passes over the upper surface of the airfoil, it will be pushed down, due to the energy provided by the treadmill motion imparted to the airflow. This apparently make the air below the airfoil denser and eventually leading to a pressure rise in the lower surface of the airfoil. Consequently, the supplemented acceleration of airflow over the upper surface of the airflow results in a greater pressure difference between both the surfaces of airfoil. Thus we are generating surplus Lift Force and minimize Drag Force by implementing Magnus Effect over the aircraft wing. We term this entire approach as Flo-Lapse. Our Pilot Studies and predictions make it seem that this idea will wide open many avenues for production of augmented Lift/Drag Ratio, wielding of shorter wingspan, probability of initiating a Vertical Take-Off for Unmanned Aerial Vehicles, or production of Lift at Zero Airspeed. A probable condition of the Cruise phase of a flight profile, where in the aircraft is at maximum speed, the incoming airstream can be used to reverse the Flo-Lapse approach and serve the purpose of an energy solution. We suggest that optimising the circumferential speed, we can have better L/D Ratios and delay early stalling of wing.
\end{abstract}

KeyWords:Magnus effect, Treadmill motion, Flo-Lapse, Aerodynamics, L/D ratio \& Aircraft wing $* * *$

\section{INTRODUCTION}

Following Anton Flettner's formulation of the behavior of rotating cylinders as Magnus Effect, portrayed as a prominent drum spinning in an airflow. With some advancements in engineering, availability of materials, development of construction of structures and getting them to work, lead the legacy of Flettner's Magnus Effect ${ }^{[1]}$. Right in the field of Aerospace and Aeronautics, engineers were interested in having a rotating cylinder as a lifting surface in contrary to the fixed wing lifting surface. There were also trials in having a combination of both rotating cylinders and fixed wing lifting surfaces. Though all of the projected theories and ideas were all seemed to be positive, there weren't many practical implementations.

Perhaps one of the first experiments on rotating cylinders dated back as early as 1925 by Reid for use in leading edge of wings. Reid ${ }^{[2]}$ reported considerable increase in lift force of rotating cylinders at various wind speeds and rotational speeds of up to $3600 \mathrm{rpm}$. In 1957, the boundary layer theory over rotating circular cylinders was developed by Glauert $^{[3]}$. In 1983, Ingham reported computational solutions of flows over rotating cylinders at very small Reynolds numbers. The aim of his studies was to assess the viscous effects over rotating cylinders. Mittal and Bhaskar ${ }^{[4]}$ have used a dimensionless spin ratio $\omega$ which was varied between 0 and 5 to study two-dimensional incompressible flows over a spinning circular cylinder at Reynolds number of 200. For high rotation rates, very large lift coefficients were observed; however, power consumed to spin the cylinders increases rapidly with spinning rate.

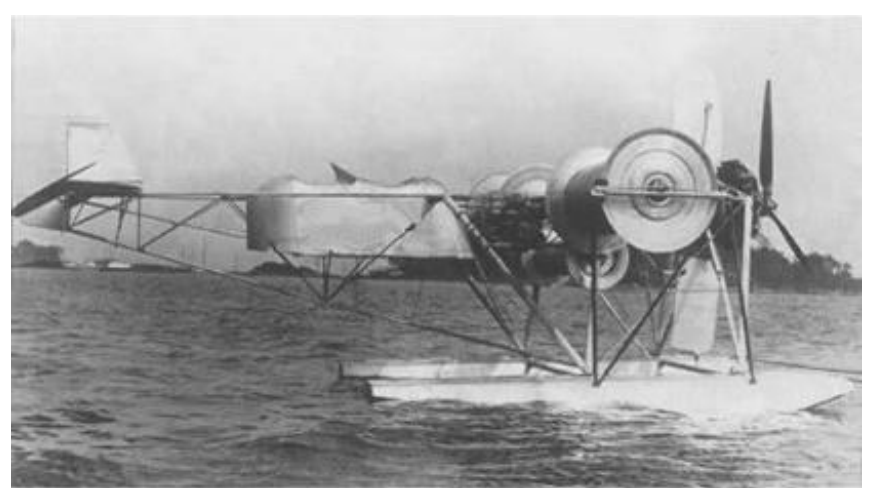

Fig-1:An aircraft uses rotating cylinders to produce lift 
In viscous steady-state flows, vorticity created at a solid surface is convected and diffused. Prandtl believed that the equilibrium state occurs at, which dictate the lift values at higher spinning rates to the maximum value of. However, experimental and numerical observations by Tokumaru and Dimotakis $^{[5]}$ suggested that the maximum lift limit can be exceeded this limit due to unsteady effects at higher aspect ratios and higher rotational speeds. Experimental and numerical works for large Reynolds numbers showed that a proper range of using Magnus effects was whilst kept drag coefficient low. However, the monotonic increase of lift coefficient over the rotating cylinders may be halted because of instabilities, 3D effects, or centrifugal forces. Some other computational evidences based on simulation of the NavierStokes equations have revealed developments of instabilities and violations from the maximum lift at high Reynolds numbers and high spin rates, simultaneously.

\subsection{Magnus Effect}

It is the commonly observed effect in which a spinning ball (or cylinder) curves away from its principal flight path. It is important in many ball sports. It affects spinning missiles, and has some engineering uses, for instance in the design of rotor ships and Flettneraeroplanes.

On a cylinder, the force due to rotation is known as KuttaJoukowski lift. It can be analyzed in terms of the vortex produced by rotation. The lift on the cylinder per unit length, $F / L$, is the product of the velocity, $V$, the density $\rho$, and the strength of the vortex that is established by the rotation, $\mathrm{G}$.

$F / L=\rho V G$

where the vortex strength is given by,

$G=2 \pi \omega r^{2}$

where $\omega$ is the angular velocity of spin of the cylinder and $r$ is the radius of the cylinder.

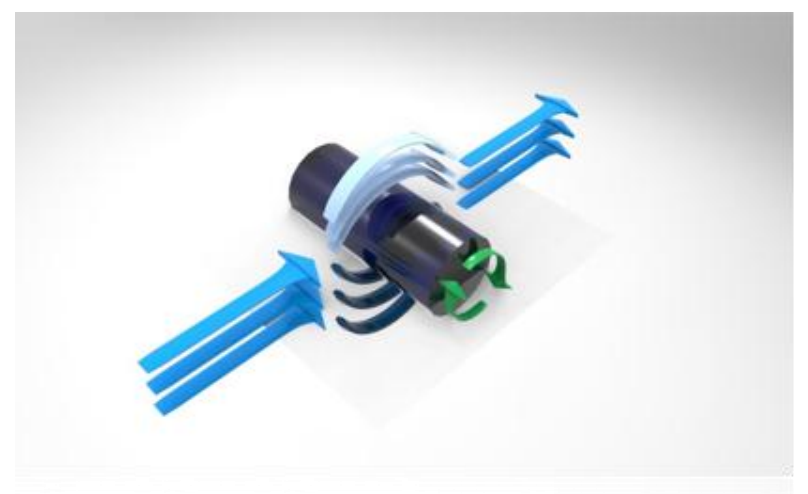

Fig-2:Flow over a rotating cylinder

Very few devices based on the Magnus effect have attracted attention or were crowned by success. On the other hand, its potential benefit compared to other airfoil-based lifting devices, such as a high lift coefficient, inspired engineers to develop devices for extracting wind energy, to propel and to steer vessels and ultimately to lift an airplane. Many research results on rotating cylinders were presented in the literature which focus mainly on the generation of aerodynamic forces.

\section{CONCEPT}

Our goal throughout the project was to implement the Magnus effect over an aircraft wing with an ambitious yet prospective view of extending the limitations of a conventional aircraft wing. A working prototype was designed, fabricated and tested.

\subsection{Insight of the model}

Our model, Flo-Lapse, is projected with an insight to have an aircraft wing fabricated with the implementation of Magnus Effect over it. We propose it to have an accelerated portion on the upper surface of the airfoil, thereby increasing the differential pressure over the upper and the lower surface of the airfoil in appreciable quantity to achieve benefits of extending the aerodynamic limitations of an aircraft wing.

The wing model for our project testing will be a symmetrical airfoil for ease of computation. It is designed with the conventional geometrical parameters to have the profile set. We will be having a rectangular cavity area in the upper surface of the wing section. There would be a moving surface, preferably a belt or a fabric of a considered co-efficient of friction.

The ultimate broken down objective would be in making the belt surface move efficiently with respective driver and driven set up of mechanical components with less complications.Our model will be driven by a small electric motor to run the treadmill set up efficiently with less power consumption, quick change of motor speed and to run at higher RPM.

\subsection{Proposed benefits}

Our concept when implemented on an aircraft after careful engineering and safety measures could be advantageous in many was which could lead to save fuel and weight. The following can be considered as advantages of wing with the use of concept Flo-Lapse.

- $\quad$ Produce more lift force with the certain wing span.

- Save cost and material used to construct lengthier wings.

- Can be used for the MUAVs (Micro Unmanned Aerial Vehicle) and UAVs (Unmanned Aerial Vehicle) for generating lift force which could lead to the possibility of VTOL (Vertical Take-off and Landing).

- Cut down the wingspan of the UAVs which are engineered to be launched in other atmospheres than earth. The transport of UAVs to another planet's atmosphere becomes challenging when the wing span is too long. 
- Reversing the concept can produce a disrupt in the flow over the wing and causes boundary layer separation which will reduce the lift produced by the wing's profile and therefore be used as a substitute to spoilers during landing.

The following CG (Computer Graphics) render produced in Autodesk Maya is conceptual and will show how the setup will look like when implemented on an Aircraft.

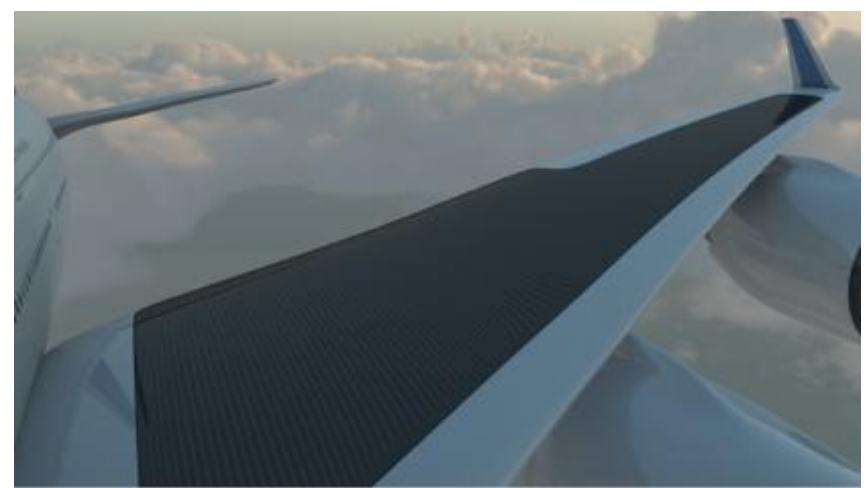

Fig-3:CG render of an aircraft with Flo-Lapse

\section{COMPONENTS OF THE MODEL}

The objective of implementing Magnus effect over our wing model is accomplished by using a Treadmill Driver assembly as mentioned in the afore topics. This chapter will involve the details about the treadmill driver assembly and their working mechanisms. Generally, a driving mechanism will involve a driver component, driven component, the assembly of moving parts such as chains or rollers or belts. The driver and components could be a pulley, gears, guides, etc.

\subsection{Driving Shafts}

In our case, we have chosen driver and driven components to be a timing pulley. The main driver component will be the shaft in the leading edge and the mail driven component will be the shaft in the trailing edge. There are holes in the stiffener for the shafts to be get through the entire wing model across its span. The shafts pass through the bearings that are specifically scouted for the designed leading and trailing edge stiffeners. These shafts are made of carbon fiber material.

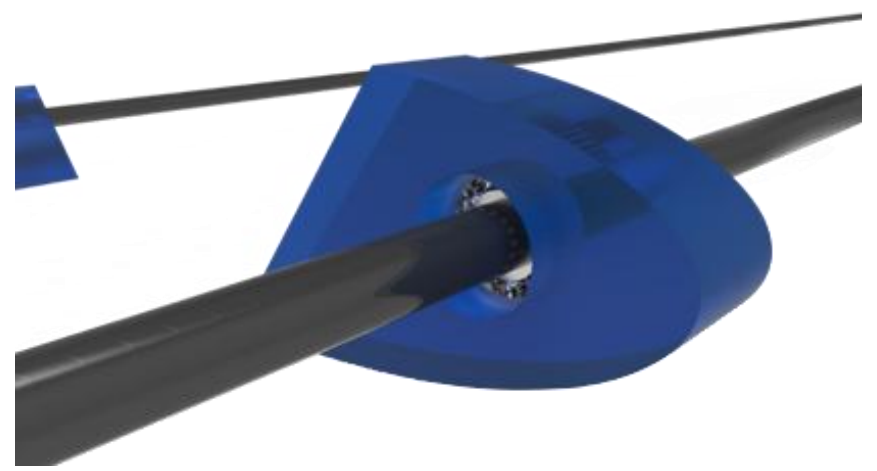

Fig-4:Stiffener housing the ball bearing \& shaft

\subsection{Timing Pulley}

There are pulley arrangements made along specific points over the driver and driven shafts. The pulleys are again passed through the shafts and are fastened with the screws to secure their positions on the shaft. They are critical components for the driving mechanism to take place. Hence the change in their position eccentricity is not preferred.

The pulley that is used for our model experiment is timing pulley. The GT2 timing pulley is used which is has 20 teeth. It is made up aluminium. Timing pulleys are as general as a normal pulley, but they have specially distributed teeth as the teeth are available in gears. These teeth are there for synchronizing the shaft, belt and pulley speed according to the time, such that thereare no slippage involved. Here, slippage is the variation of speed of all the rotating components and are not synchronized properly.

The timing pulleys are positioned along the driver and driven shafts with respective intervals marked on them.

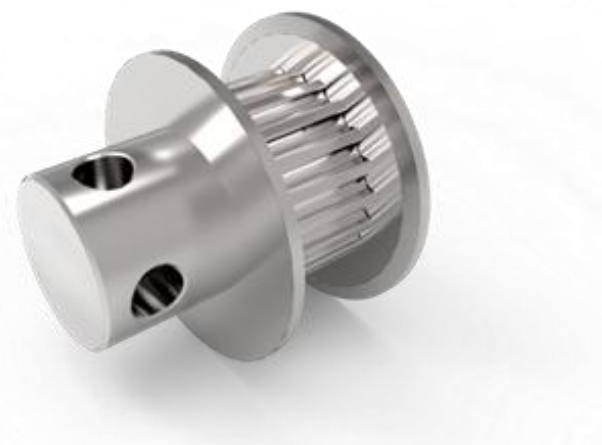

Fig-5:Timing Pulley

\subsection{Timing Belt}

Over these timing pulleys, the timing belts are made to run. The main belt that runs along the cavity area of the wing section. The inwards side of the main belt has the timing belt embedded on the points along the cavity area span to run over the timing pulley to have even minimal slippage or no slippage. This way we achieve as much as efficient treadmill movement in our wing model.

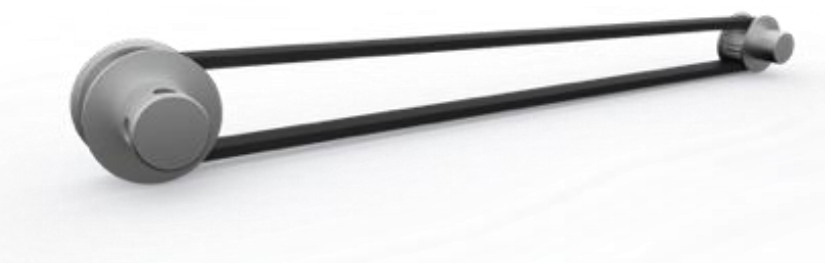

Fig-6:Timing pulley and belt setup

\subsection{Driver Setup}

The driver setup is nothing but a Brushless DC Motor coupled with a flexible coupling to the treadmill driver shaft assembly. The Brushless DC Motor is controlled by an electronic speed controller, which receives Pulse Width Module Signals from a micro-controller that we use for our project. 


\subsection{Motor}

Brushless DC Motor are also called Electronically Commutated Motors. They are synchronous motors that are powered by a DC Electric Source via an switching power supply to produce an AC Electric signal to drive the motor. The rotor part of a brushless DC motor is a permanent magnet around an armature, coils with a number of windings. The rotor set up picks up synchronized electric signals from the Electronic Speed Controller. This controller requires some means of determining the rotor orientation and position. It contains a 3 Directional outputs. These outputs are controlled by a logic circuit. The motor has a $\mathrm{KV}$ rating of 1000 and can be used with a LiPo battery which has 3 cells in series (11.1V).

\section{DESIGN CALCULATIONS}

\subsection{Timing Pulley \& Belt}

\section{a. Gear ratio}

The timing pulley $\&$ belt have teeth on them which are similar to gears. The timing pulley is placed on the front shaft and belt is stuck to the rear shaft to form a small gear around it. Therefore, in order to meet the design requirements, Gear ratio should be known. The gear ratio of a gear train, also known as its speed ratio, is the ratio of the angular velocity of the input gear to the angular velocity of the output gear. The gear ratio can be calculated directly from the numbers of teeth on the gears in the gear train. The torque ratio of the gear train, also known as its mechanical advantage, is determined by the gear ratio.

The number of teeth of the driver gear $=20$

The number of teeth of the driven gear $=7$

$R=7 / 20=0.35$

When $\mathrm{R}$ is lower than 1 , the rear shaft will be able to carry out more speed than the front shaft. When the gear ratio is equal to 1 , then the torque and speed transmitted will be equal in the both the shafts. $\mathrm{R}<1$ is considered in our design to enable movement of the belt effectively.

\section{b. Length of the belt}

For calculating the length of the moving belt, the diameter of the front and rear shaft is required along with the distance between both the shafts.

The length of the belt is calculated with the following formulae,

\section{Length of the belt $=d_{m} \pi / 2+\left(d_{f} \pi / 2\right)+2 l_{f m}+\left(d_{f^{-}} d_{m}\right)^{2} / 4 l_{f m}$}

\section{Where}

$d_{m}$ is the diameter of the driven shaft $=5 \mathrm{~mm}$,

$d_{f}$ is the diameter of the driver shaft $=3 \mathrm{~mm}$,

$1_{\mathrm{fm}}$ is the distance between both the centers of the shafts = $99.881 \mathrm{~mm}$ (from CATIA).

By substituting the values in the formulae,

Length of the belt $=212 \mathrm{~mm}$

\section{c. Velocity of the belt}

The velocity of the belt if calculated with $d_{m}$ the diameter of the rear shaft, $\mathrm{n}_{\mathrm{m}}$ the RPM of the motor in use and the following formulae.

$V=\pi d_{m} n_{m} / 25.4$ (mm/Minute)

For finding the RPM of the motor in use, the KV rating of the motor and the voltage supplied to it are substituted in following formulae.

$n_{m}=K V \times V=1,000 \times 12=12,000 R P M$

For safety concerns, maximum of only $50 \%$ of throttle was considered during testing and therefore,

$\mathrm{n}_{\mathrm{m}}=6,000$ RPM

Now substituting the available values in the formulae to find the velocity of the belt,

$V=2225.196 \mathrm{~mm} /$ Minute $=37.08 \mathrm{~m} / \mathrm{s}$

The maximum velocity of the belt will be $37.08 \mathrm{~m} / \mathrm{s}$ without considering lose due to friction.

\subsection{Wing section}

The parameters of the wing section were carefully chosen to ensure that the wing section can be placed for testing in the test section of the subsonic wind tunnel available in our University. But later on due to complications of using the force balance in the wind tunnel, we tested using the Cascade wing tunnel that was available.

The following are the parameter of the wing section.

- $\quad$ Span of the wing section (b) $=400 \mathrm{~mm}=0.4 \mathrm{~m}$

- Chord of the wing section (c) $=150 \mathrm{~mm}=0.15 \mathrm{~m}$

- $\quad$ Aspect ratio $(\mathrm{AR})=2.667$

- $\quad$ Airfoil used = NACA 0021

- $\quad$ Type of airfoil = Symmetrical

Symmetrical airfoil generates no Lift force at an angle of attack of $0^{\circ}$ therefore is not used in many applications in today's scenario instead, cambered airfoils are preferred. We chose NACA 0021 to prove that Lift can be generated with symmetrical airfoil when our concept is introduced to it. So imagine the possibilities and the lift values that can be obtained from a cambered airfoil when this concept is applied to them. Moreover, NACA 0021 has a maximum thickness of about $21 \%$ of the chord length which accounts to about $31.5 \mathrm{~mm}$ that was required to house the components in this small scale. But the proposed idea can be applied to any airfoil type and it only depends on the ways it is engineered.

Surface area $(S)=b^{2} / A R=0.0601 \mathrm{~m}^{2}$

Thickness of the wing section $=3 \mathrm{~mm}$

Cavity length (Spanwise) $=300 \mathrm{~mm}=0.3 \mathrm{~m}$

Cavity breadth (Chordwise) $=90 \mathrm{~mm}=0.9 \mathrm{~m}$

Cavity area $=0.3 \times 0.9=0.27 \mathrm{~m}^{2}$

The cavity area starts from $25 \mathrm{~mm}$ from the leading edge and ends at $115 \mathrm{~mm}$ from the leading edge measured along the chord. These values were taken after careful consideration and analysis with ANSYS Fluent of the 3D model designed in CATIA V5. $50 \mathrm{~mm}$ allowance was given 
on both sides spanwise of the cavity area to house all of the internal components.

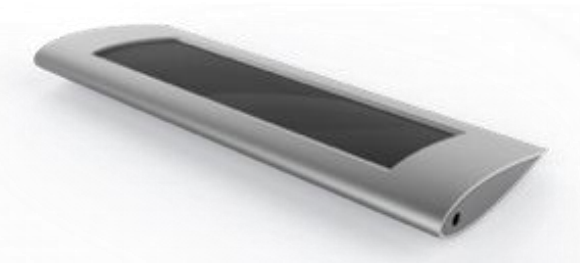

Fig-7:CG render of Flo-Lapse wing section

Vortex panel method can be considered for doing the theoretical calculations and to find the lift force produced by the wing section. Prandtl lifting-line theory was an alternative approach to do find the lift, but can be applied only for thin airfoils which have a thickness less than $12 \%$ of the chord. The Vortex panel method, approximates the airfoil by using a series of infinite, discrete bound vortices to approximate a continuous distribution of vorticity. In the unsteady case, the airfoil wake is similarly approximated. As well, since all motions considered are planar, a 2dimensional approximation was employed. But the theoretical calculations can be difficult when it comes to prove this concept as there is a moving surface involved to produce the lift. A lot of approximations need to be considered and will lead to miscalculations. Powerful CFD package like ANSYS Fluent and CFX were considered over theoretical calculations to save time and to precisely obtain values by solving complex equations.

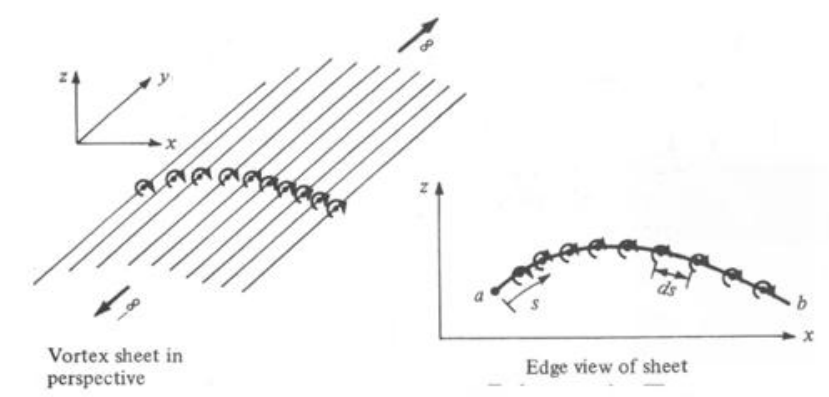

Fig-8:Vortex panel method

\subsection{Detailed design}

We designed every component in the CAD package, CATIA V5. Designing these components helped us and the professors to understand the assembly and structure of the concept with precision even before it is fabricated. The 3D designing process in CATIA V5 was similar in almost all cases and were really necessary to be precise with the design.

Stiffeners were required to keep the hollow surface intact and to house the ball bearings. The stiffeners were designed to fit the curvature of the wing profile. They were placed in 4 key regions, two in the front and two in the rear part of the wing section. A hole with the outer diameter of the available radial ball bearing was made so that it will sit perfectly inside it. The outer diameter of the ball bearing used in front of the wing was $10 \mathrm{~mm}$ and that placed in the rear wing was $6 \mathrm{~mm}$. The thickness of the stiffener is $10 \mathrm{~mm}$.

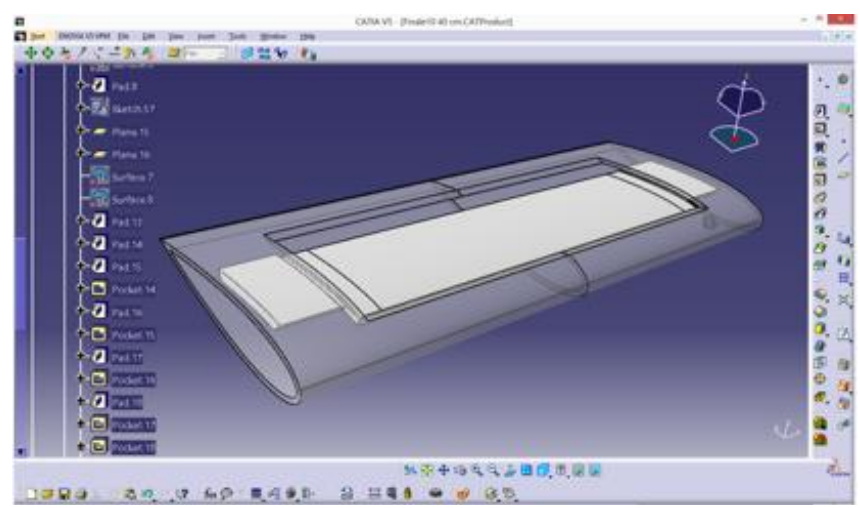

Fig-9:Wing section designed in CATIA V5

The final assembly was done with the help of the product workbench available in CATIA V5. The stiffener will contain the radial ball bearings and the ball bearing will hold the shafts in place without restricting the radial motion of the shafts. Coincidence constraint option available in the software was used for this purpose. The required contact and surface constraints have been made to precisely see and understand what the final product will look like and some adjustments were made accordingly. After assembly was complete, the file was exported from CATIA and then imported into Autodesk Maya for a final render. After everything seemed to be right, the components like the ball bearing, flexible coupler, motor, ESC, battery, timing pulley and belt which need to be bought were immediately procured and were kept ready so that the physical assembly of the model can be made after obtaining the $3 \mathrm{D}$ printed model.

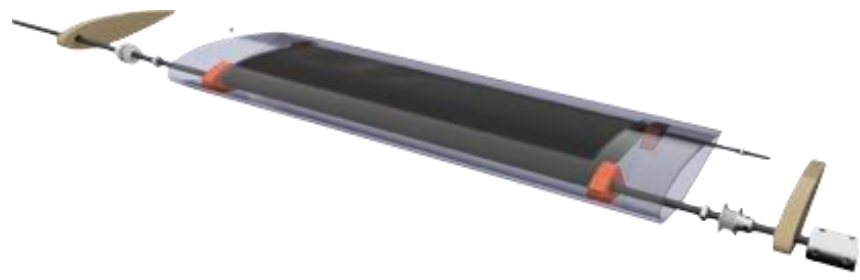

Fig-10:CG render Flo-Lapse wing section

\section{COMPUTATUINAL FLUID DYNAMICS}

We used Ansys Fluent and CFX to perform a CFD analysis of the structure. We performed 3D analysis in CFX for optimising the wing structure for minimum drag and performed 2D analysis in Fluent to provide results for the concept.

Table-1:Comparison for optimization

\begin{tabular}{|l|l|}
\hline Wing section $(\mathbf{4 0 c m})$ & Drag $(\mathbf{N})$ \\
\hline NACA 0021 & $\mathbf{1 . 0 4 6 0}$ \\
\hline Flo-Lapse Original & $\mathbf{1 . 5 4 5 4}$ \\
\hline Flo-Lapse Optimised & $\mathbf{1 . 1 3 3 4}$ \\
\hline
\end{tabular}


The results obtained were really positive and showed that lift can be generated. The following results were calculated with a constant air flow velocity of $35 \mathrm{~m} / \mathrm{s}$ since the cascade wind tunnel in use for the experimental test will produce a maximum of $35 \mathrm{~m} / \mathrm{s}$ of flow. Different belt velocities were analysed for Lift and Drag forces and were included in the results section. The maximum possible belt velocity of about $35 \mathrm{~m} / \mathrm{s}$ (calculated theoretically considering the potential of the motor and keeping a safe limit) is considered in this section keeping the angle of attack at $0^{\circ}$.

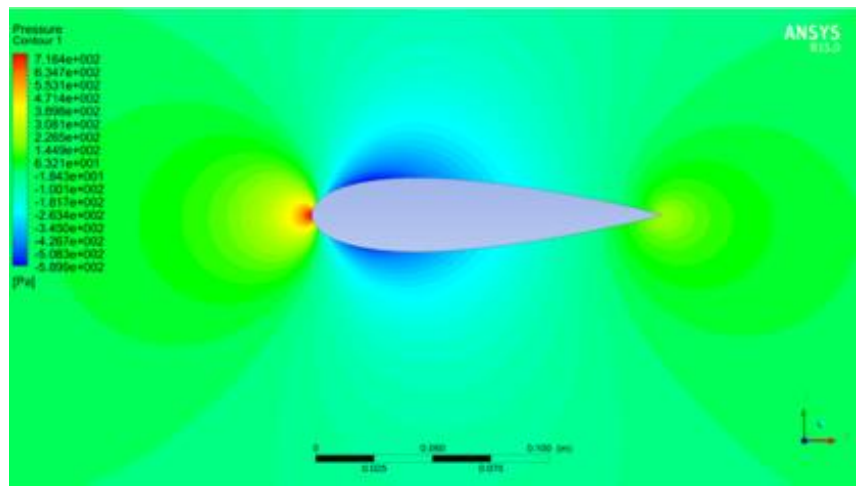

Fig-11:Contour plot of Pressure for concept Flo-Lapse

Theoretically, there will not be any lift force generated from the wing section when there is no moving surface. But with the moving surface, the symmetrical airfoil produces a Lift force of 8.088 Newton per unit wing span or 0.824 Kilogram per unit wing span and a drag force of about 1.895 Newton per unit wing span.

The above contour plot for pressure, clearly shows that the pressure on the upper surface of the symmetrical airfoil is lesser than that of the lower surface. Since a pressure difference is observed, there is a lift force acting on the body which tends to prove the concept valid. We observed that the pressure keeps on decreasing when the belt velocity is increased to higher levels. We also noticed that the Drag value decreased on the increase of the belt speed which added value to the concept as well. The favourable decrease in drag value is because of the reduction in skin friction and due to delayed boundary layer separation.

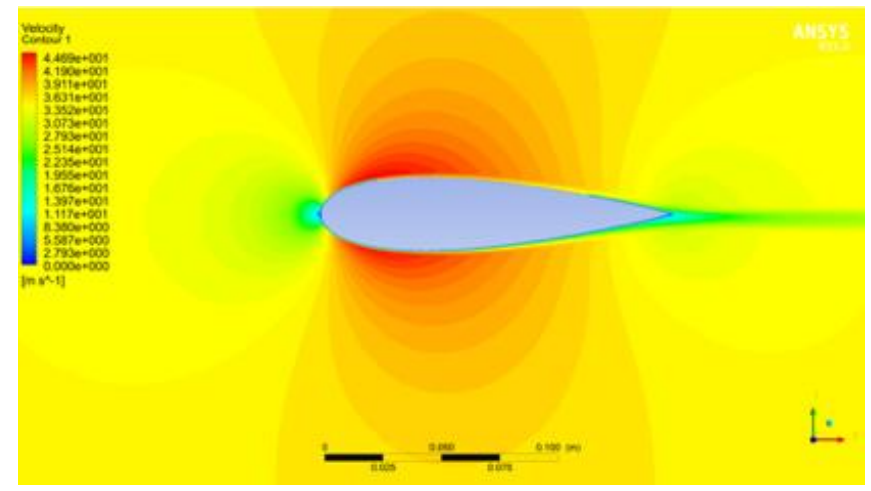

Fig-12:Contour plot of velocity for concept Flo-Lapse

The above image shows the contour plot for velocity in $\mathrm{m} / \mathrm{s}$. It is observed that the velocity is slightly higher on the upper surface of the wing than the lower surface which can seen from the colour map. The belt moving in a clockwise direction, pushes the air in the direction of the air flow, imparting some energy to the air particles. By Bernoulli's principle, when velocity increases, the pressure decreases which causes a difference in pressure and ultimately lift is produced.

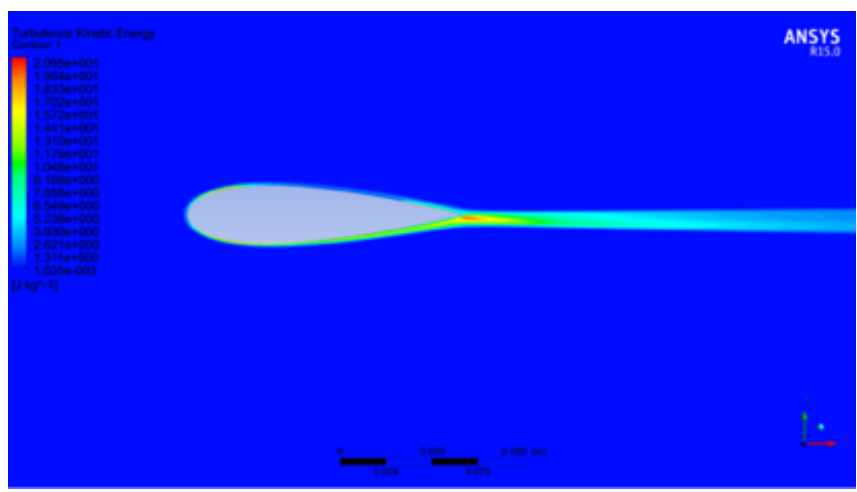

Fig-13:Contour plot of Turbulence Kinetic Energy for concept Flo-Lapse

The Turbulence Kinetic Energy (TKE) is the mean kinetic energy per unit mass associated with eddies in turbulent flow. By looking at the contour plot for TKE, it can seen that the kinetic energy imparted due to turbulence is less in region where the moving surface is installed in and increases after the moving region. This also proves to be a positive result for the concept and the project.

\section{FABRICATION (3D PRINTING)}

$3 \mathrm{D}$ printing or additive manufacturing is a process of making three dimensional solid objects from a digital file. The creation of a 3D printed object is achieved using additive processes. In an additive process an object is created by laying down successive layers of material until the entire object is created. Each of these layers can be seen as a thinly sliced horizontal cross-section of the eventual object.Not all 3D printers use the same technology to realize their objects. Some methods use melting or softening material to produce the layers. Selective laser sintering (SLS) and fused deposition modeling (FDM) are the most common technologies using this way of printing. Another method of printing is to lay liquid materials that are cured with different technologies. The most common technology using this method is called stereolithography (SLA).

The virtual $3 \mathrm{D}$ design that needs to be manufactured is created using a CAD software. We chose Dassault Systemes CATIA V5. The part is carefully designed with atmost precision to completely utilise the 3D printer's capabilities. To prepare the digital file created in CATIA for printing, the file should be exported in STL format and then imported to the printer's software. Then software slices the final model into hundreds or thousands of horizontal layers. When this prepared file is uploaded in the $3 \mathrm{D}$ printer, the printer creates the object layer by layer. The 3D printer reads every slice (or 2D image) and proceeds to create the object blending each layer together with no sign of the layering visible, resulting in one three dimensional object. 
Before printing a 3D model from an STL file, it must first be examined for "manifold errors," this step being called the "fixup." Especially STLs that have been produced from a model obtained through 3D scanning often have many manifold errors in them that need to be fixed. Examples of manifold errors are surfaces that do not connect, or gaps in the models. Examples of software that can be used to fix these errors are netfabb and Meshmixer, or even Cura, or Slic3r.

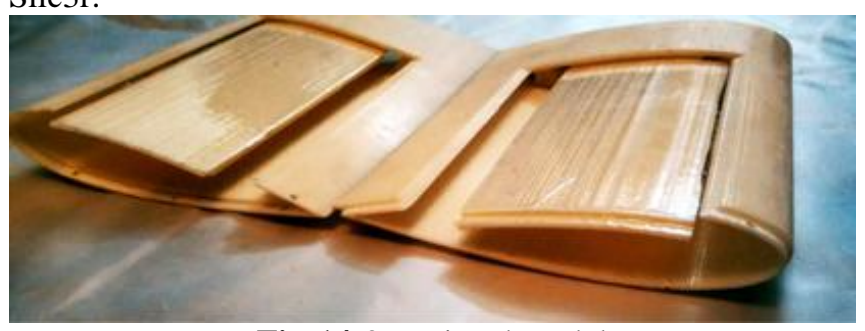

Fig-14:3D printed model

The time that the printer took for completing the $3 \mathrm{D}$ printed wing section of about $400 \mathrm{~mm}$ with a precision of upto 0.1 $\mathrm{mm}$ was about 22 hours. The final product came out really well as expected. Attention given to minute details and precision were well received and printed accordingly. It was an absolute joy to hold the 3D design which we have designed which looked brilliant.

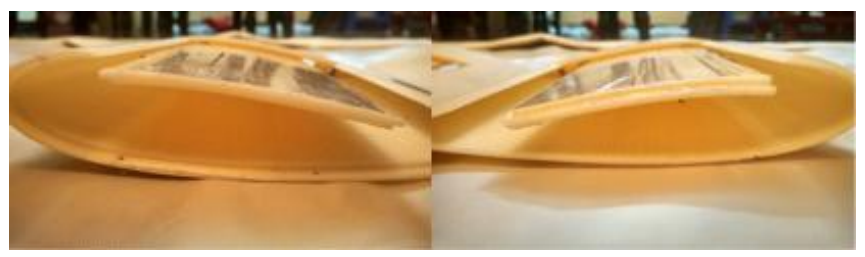

Fig-15:Side view of the wing section

\section{TESTING}

In order to validate the concept of implementing Magnus Effect into an aircraft wing, we need to follow a strict testing scheme adhering to our technical objective by calculating the aerodynamic forces over this Magnus Wing. The test requirement is to have the model running in real time with a dynamic test in a wind tunnel and measure the aerodynamic forces generated by the model.Our interest is in determining the primary aerodynamic forces: Lift and Drag Force.

Apart from this, the awakened by product of our proposed idea will actually delay the boundary layer separation over the upper side of the wing and the boundary layer thickness will be reduced in an appreciable scope of length.As we are testing our run towards the primary objective of determining the aerodynamic forces around the model, we are also interested in determining the flow of the pressure at a particular point along the chord of the wing in the upper surface of the wing model. The pressure values are noted at elevated distances from the wing model upper side.

We are conducting the tests in our SRM University Aerospace Engineering Hangar. Our Hangar is spacious and many of the test equipments are available for student's projects and interest of studies. We are making use of a cascade wind tunnel available in our department hangar. It is capable of running at 100 Kilometers per Hour , nearly 30 $\mathrm{m} / \mathrm{s}$. It was designed and fabricated our university passed out aerospace engineering students.

\subsection{Force Balance Rig}

Our main criteria in making our Force Balance Apparatus is to determine the Lift and Drag forces with an ease of measurement. We will be building this Simple 2 component Force Balance Test Rig with wood material of $2.54 \mathrm{~cm}$ thickness.

\section{a.Weighing Arm}

The Arm is $60 \mathrm{~cm}$ in length, the leading edge of the arm is at the distance of $45 \mathrm{~cm}$ from the center and the trailing edge of the arm.

The weight hangars are hooked at both the edges of the weighing arm.

\section{b.Support Stem}

The support stem is of $57.5 \mathrm{~cm}$ length. It adjoins the center point of the weighing arm with an hinged support to have a see-saw motion of the weighing arm as per the forces generated by the model due to wind speed from the wind tunnel flow.

\section{c.Wing Stem}

The stem is inclined at an angle from the leading edge of the weighing arm. At the top portion of the wing stem is the support for wing to be fastened for testing. The length of the wing stem is about $35 \mathrm{~cm}$.

\section{d.Motor Frame}

The motor frame is actually a $U$ shaped frame, on the same axis as the wing stem. The main purpose of the motor frame is not to constrain the motion of the wing if the motor is fastened to one fixed surface, it is of very much need that the motor follows the curve profile made by the wing model during test due to the aerodynamic forces. Hence there wouldn't be any hindrances for the motion of either the motor or the wing model.

\section{e.Weight Hanger}

The weight hangars are hooked to the edges of the weighing arms of the Force Balance rig apparatus.

\section{f.Weight Pans}

The weight pans are hung with both the weight hangers.

\section{g.Known Weights}

Few known weights are placed over the weight pans, as they are the measured values for the forces.

\section{h.Angle of Attack Knobs}

We have to test the model in different angles of attack for a certain speed, so it was ideal to have a manual knob to set the desired angle of attack. 


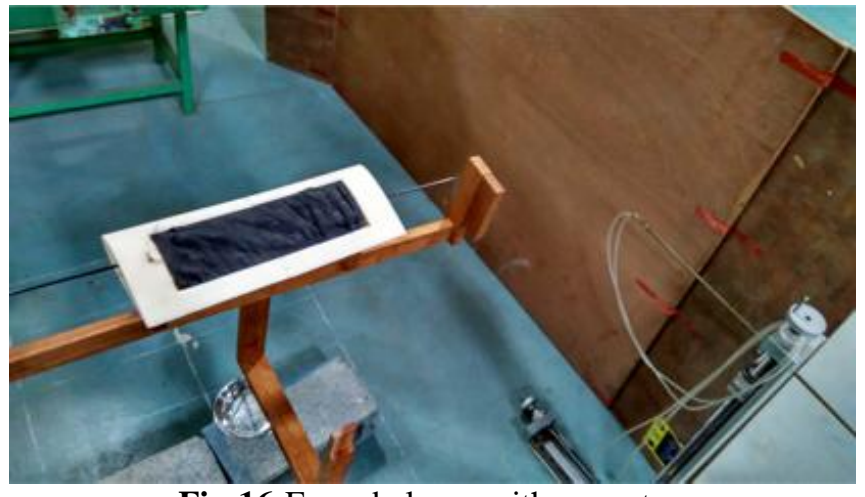

Fig-16:Force balance with our setup

\section{RESULTS}

The lift and drag forces were measured and tabulated.

Table-2: Velocity of air flow at $30 \mathrm{~m} / \mathrm{s}$

\begin{tabular}{|l|l|l|l|l|}
\hline $\begin{array}{l}\text { Speed of } \\
\text { the motor }\end{array}$ & $\begin{array}{l}\text { Angle of } \\
\text { Attack } \\
\text { Degrees })\end{array}$ & $\begin{array}{l}\text { Lift } \\
(\mathbf{N})\end{array}$ & $\begin{array}{l}\text { Drag } \\
(\mathbf{N})\end{array}$ & L/D \\
\hline $\mathbf{0 \%}$ & 0 & 0.017 & 1.813 & 0.010 \\
\hline $\mathbf{2 5 \%}$ & 0 & 3.488 & 1.503 & 2.321 \\
\hline $\mathbf{5 0 \%}$ & 0 & 6.007 & 1.363 & 4.407 \\
\hline $\mathbf{0 \%}$ & 5 & 17.445 & 1.014 & 17.202 \\
\hline $\mathbf{2 5 \%}$ & 5 & 22.519 & 0.468 & 48.157 \\
\hline $\mathbf{5 0 \%}$ & 5 & 26.367 & 0.156 & 168.823 \\
\hline
\end{tabular}

It can be observed from the tabulation that the Lift increases as the speed of the upper surface of the wing increases. Due to safety reasons, the motor speed was limited and the concept was tested within the limits to protect the model.

Table-3: Velocity of air flow at $35 \mathrm{~m} / \mathrm{s}$

\begin{tabular}{|l|l|l|l|l|}
\hline $\begin{array}{l}\text { Speed of } \\
\text { the motor }\end{array}$ & $\begin{array}{l}\text { Angle of } \\
\text { Attack } \\
\text { (Degrees) }\end{array}$ & $\begin{array}{l}\text { Lift } \\
(\mathbf{N})\end{array}$ & $\begin{array}{l}\text { Drag } \\
(\mathbf{N})\end{array}$ & L/D \\
\hline $\mathbf{0 \%}$ & 0 & 0.022 & 2.401 & 0.009 \\
\hline $\mathbf{2 5 \%}$ & 0 & 4.130 & 2.033 & 2.032 \\
\hline $\mathbf{5 0 \%}$ & 0 & 7.430 & 1.845 & 4.027 \\
\hline $\mathbf{0 \%}$ & 5 & 23.780 & 1.306 & 18.207 \\
\hline $\mathbf{2 5 \%}$ & 5 & 29.534 & 0.669 & 44.118 \\
\hline $\mathbf{5 0 \%}$ & 5 & 34.422 & 0.267 & 128.877 \\
\hline
\end{tabular}

The following graph plots clearly illustrate that the L/D ratio increases with the increase in the motor speeds.

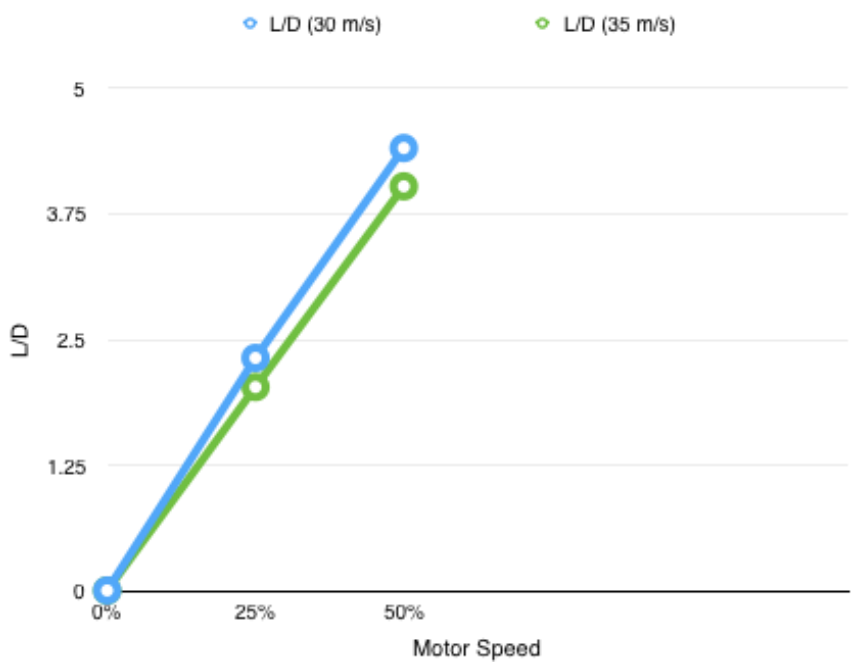

Chart-1:Graph between Motor speed and L/D at an AOA of $0^{\circ}$

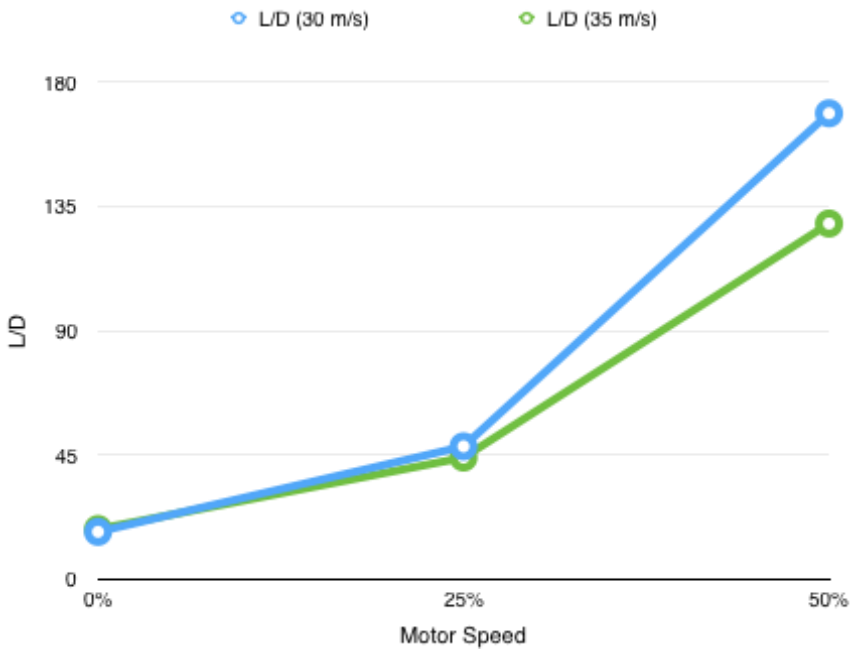

Chart-2:Graph between Motor speed and L/D at an AOA of $5^{\circ}$

\subsection{Scope for improvement}

The results that we have acquired from the CFD and Laboratory testing have resulted in a positive way validating all our postulates and helping our proposed idea to be authenticated with the values.

We still believe, there are lots of scopes left in this topic to be explored and studied in depth. We feel, having better laboratory testing equipment such as strain gauge force balance equipment will be of great help in finding precise values of aerodynamic forces acting on the body.In the analysis front, scopes of UDF for making the belt move in $3 \mathrm{D}$ space along the contour of the wing section is a tedious task but can be utilized for precise calculations and analysis. UDF or User Defined Functions is used in dynamic meshing. 
The main idea of this concept arouse to shorten the wing span of the drones that will be manufactured in future for surveillance or other space missions. It was found from CFD that planets with denser atmosphere than Earth will be benefited the most out of this concept.

\section{Conclusion}

The results that we have obtained from $\mathrm{CFD}$ and experimental study show that the concept is valid and can be used to generate a far higher values of Lift force and also decrease the Drag force that is obtained from a wing section. This opens up a new category and endless possibilities to research on and benefit from them. Further studies and tests should be made on this topic to prove the delay in boundary layer separation.

The major advantages of a Magnus effect device are highlift forces or rather high wing-loading and stall resistance. The disadvantages are the need for an additional driving mechanism with additional weight and complexity compared to a conventional wing. From a technical point of view, there are some mature Magnus effect devices available that can enhance the high-lift capability of a STOL aircraft or even the aerodynamic efficiency of a conventional aircraft.

Many ideas exist on how to use the Magnus effect. Perhaps, in a while concept Flo-Lapse can be seen integrated in future UAVs or aircrafts.

\section{ACKNOWLEDGEMENT}

The authors would like to acknowledge the technical help of Prof. Mohamed Arif, Prof.Karuppudaiyan, Prof. M. Umar Rizwan,Mr.Ramesh and Mr.S.Jaichandran.

\section{REFERENCES}

[1].Seifert, J., A review of the Magnus effect in aeronautics. Progress in Aerospace Sciences (2012); 55:17-45.

[2].Reid EG. Tests of rotating cylinders. Flight; 1925.

[3].Glauert,M.B.,A boundary layer theorem with applications to rotating cylinders.J.Fluid Mech.; 1957:2:89.

[4].Mittal, S., Kumar, B., Flow past a rotating cylinder. Journal of Fluid Mechanics; 2003:476:303-334.

[5].Tokumaru, P., Dimotakis, P., Rotary oscillation control of a cylinder wake. J. Fluid mech.; 1991:224:77-90.

\section{BIOGRAPHIES}

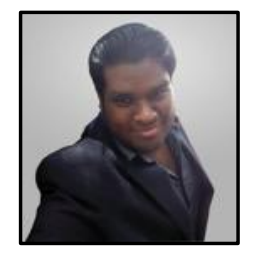

KavithasanPatkunam is a graduate in Aerospace Engineering from SRM University, India. Currently pursuing Masters Studies in Aerospace Engineering in KTH Royal Institute of Technology, Sweden.

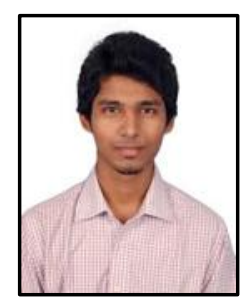

SamaySigamani is a graduate in Aerospace Engineering from SRM University, India. Currently pursuing Masters Studies in Air Transport Operations in ÉcoleNationale de l'AviationCivile, France.

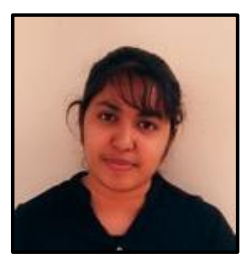

PedaballiMahathi is a graduate in Aerospace Engineering from SRM University, India.

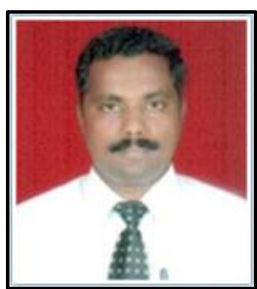

MrSelvakumaran $\mathrm{T}$ is currently a Senior Grade Assistant Professor in SRM University, lecturing in Solid Mechanics, Aircraft Structures and Engineering Graphics. He's also a Lifetime member in the Aeronautical Society of India. 